GENETICS AND PLANT BREEDING

\title{
Genetic Analysis of Yield and Yield Contributing Traits in Bread Wheat
}

\author{
Anjali Joshi*, Anil Kumar and Sakshi Kashyap \\ Department of Genetics and Plant Breeding, College of Agriculture, Govind Ballabh Pant University of Agriculture and Technology, \\ Pantnagar, U.S. Nagar, 263145, Uttarakhand, India
}

"Corresponding author: anjali999aj@gmail.com (ORCID ID: 0000-0002-7841-2326)

Paper No. 826

Received: $14-01-2020$

Revised: 17-04-2020

Accepted: 27-05-2020

\begin{abstract}
Ten wheat genotypes were crossed in diallel manner excluding reciprocals. The crosses along with parents were analysed for combining ability and significant variation was found amongst genotypes for various yield and yield contributing traits. ANOVA revealed that mean square for general combining ability was significant for all the traits studied except for spike length. Mean square for SCA was also non significant for spike length while for all other characters it was significant. Out of total 45 crosses 5, 8, 4, 7, 8, 3, 3, 11, $11,13,8$ and 11 crosses showed significant SCA effects in desirable direction for days to $75 \%$ heading, days to maturity, flag leaf area, number of productive tillers per plant, plant height, spike length, number of spikelets/spike, number of grains/ spike, 1000 grain weight, biological yield/plant, grain yield/plant and harvest index, respectively. Two cross combinations viz., HD $2967 \times$ NIAW 1594 and KFA/2*KACHU × WH 1187 were identified as good crosses due to them having higher per se performance and significant SCA effects for three yield and yield contributing traits so these crosses presents an opportunity for commercial exploitation either in form of hybrid varieties or as base material for selection of potential homozygous lines from transgressive segregants for improvement of yield levels of bread wheat.

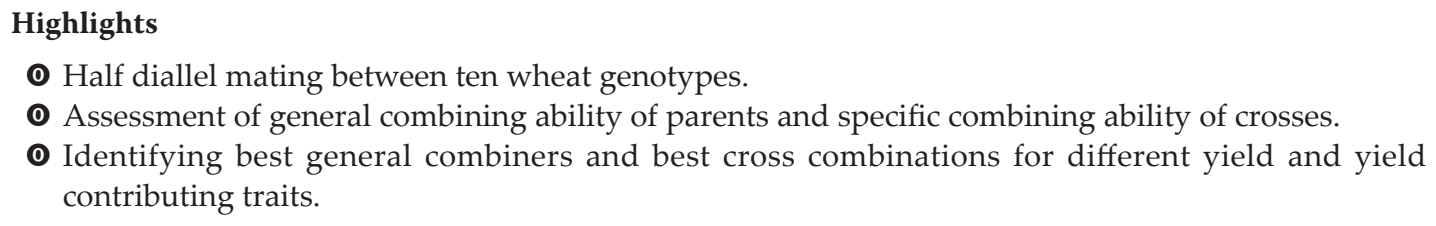

Keywords: wheat, combining ability, GCA, SCA, gene action

Bread wheat (Triticum aestivum L. em. Thell) is an allohexaploid $(2 n=6 x=42, A A B B D D)$ originally belonging to the Levant region. It is widely cultivated throughout the world and covers an area of 220.40 million hectares globally with a production of 729.01 million tonnes and $3307.4 \mathrm{~kg} /$ ha productivity (FAO, 2014). On a global level it acts as a major provider of digestible sugar contributing $55 \%$ carbohydrate and $20 \%$ of the total food calories (Breiman and Graur 1995). The highest wheat production in world comes from China followed by India. Under Indian context wheat is an important staple food crop for many states and is grown over an area of 20.23 million hectares contributing 30\% to the Indian food basket (Anonymous 2016). Being one of the most important crop of the rabi season in India wheat gives a mammoth production of 93.50 million tonne and $3093 \mathrm{~kg} / \mathrm{ha}$ productivity. The major wheat producing states in India are Uttar Pradesh (26.9 million tonne), Punjab (16.11 million tonne) and Haryana (11.14 million tonne).

In India three wheat species are cultivated i.e., Triticum aestioum, T. durum and T. dicoccum. The largest area under wheat cultivation i.e., 95\% is occupied by Triticum aestivum also called as common wheat or bread wheat. It is grown in all 
six agroclimatic zones of the country viz., Northern Hill Zone (NHZ), North West Plain Zone (NWPZ), North East Plain Zone (NEPZ), Central Zone (CZ), Peninsular Zone (PZ) and Southern Hill Zone (SHZ) and is used for a variety for purpose including making of bread, biscuit, chapatti, cookies, noodles, cakes etc. Four percent area under wheat production in India is occupied by T. durum or macroni wheat. It is adapted to Central and Peninsular Zone and used for production of macaroni, vermicelli and spaghetti. T. dicoccum covers only $1 \%$ area of India and is grown only in Peninsular Zone of the country and is used for making chapatti, macaroni, spaghetti and also has medicinal value.

Wheat is a staple food crop for nearly two billion people i.e., $36 \%$ of the world population. With the increase in population the demand of wheat is increasing continuously (Joshi et al. 2018b). India is expected to surpass China by the year 2022 in terms of number of individual residing in the country, with its population reaching 1.7 billion by 2050 . The production of wheat is 93.50 million tonnes in 20152016 . With a population growth rate of $1.2 \%$, the production of wheat must increase at the rate of 1.8 percent per year to meet the demand of increasing population. By the year 2035 the production must increase by 42 million tonnes. Global demand for wheat is growing at approximately $2 \%$ per year, twice the current rate of gain in genetic yield potential (Skovmand and Reynolds 2000). The first tremendous increase in wheat production from 11 million tons in 1966-67 to 17 million tons in 1967-68 owing to the influx of quality seeds, new equipment and technologies, new methods for production was the driver of green revolution in India. Further increase in production of wheat is only possible by increasing the production per unit area of wheat as further increase in area under wheat cultivation is not possible due to limited land and the production practices of wheat have already been optimized. Imrovement of wheat quality is also an important need of present times (Joshi et al. 2018a, Joshi et al. 2019 and Joshi et al. 2020). For yield enhancement input responsive and high yielding varieties must be produced. In order to achieve this target it is essential to know the type of gene action involved in controlling different yield contributing traits of wheat so as to determine the required breeding programme in order to breed superior varieties. Also for achieving improvement by means of hybridisation identification of good general combiner and superior cross combinations is necessary. Combining ability analysis developed by Griffing (1956) provides a clear idea about the performance of the $F_{1}$ developed by them and is helpful in choosing of superior parents for hybridisation programme. Therefore present investigation was conducted to identify suitable parents for hybridization on the basis of combining ability and per se performance and also to determine superior cross combinations for various yield and yield contributing traits which can be used for isolation of superior transgressive segregants and development of improved variety to further enhance food security.

\section{MATERIAL AND METHODS}

The experiment was conducted at Norman E. Borlaug Crop Research Centre, Govind Ballabh Pant University of Agriculture and Technology, Pantnagar, India. Crossing of 10 parental lines namely, KAUZ/ALTAR84/3/MILAN/KAUZ/4/ HUITES, QLD 39, UP 2762, KFA/2*KACHU, RAJ 4419, PBW 729, WH 1187, HD 2967, DBW 50 and NIAW 1594 in half diallel fashion excluding reciprocals to produce 45 hybrids was done during Rabi 2015-16 and the F1 progenies generated along with parental lines and checks (UP 2628 and WH 1105) were planted for evaluation during Rabi 2016-17 in randomized block design with three replications. The row to row spacing was $20 \mathrm{~cm}$ and plant to plant distance was $10 \mathrm{~cm}$. Each entry was planted in two rows of $1 \mathrm{~m}$ in each replication.

Observation was recorded for twelve different yield and yield contributing traits as Days to 75\% heading, Days to maturity, Flag leaf area $\left(\mathrm{cm}^{2}\right)$, Plant height at maturity $(\mathrm{cm})$, Number of productive tillers/plant, Spike length (cm), Number of spikelets/ spike, Number of grains/spike, 1000 grain weight, Biological yield/plant, Grain yield/plant and Harvest index (HI). For characters as Days to 75\% heading, Days to maturity, Flag leaf area $\left(\mathrm{cm}^{2}\right)$, Plant height at maturity $(\mathrm{cm})$, Number of productive tillers/ plant, Spike length $(\mathrm{cm})$, Number of spikelets/ spike, Number of grains/spike, 1000 grain weight, Biological yield/plant, Grain yield/plant observations were taken on five randomly selected competitive plants for each entry. The mean value of five plants 
was used for analysis. While observations were taken on whole plot basis for characters as days to $75 \%$ heading and days to maturity. Harvest index for each entry was calculated by utilising the value of biological yield/plant and grain yield/plant as per the following formula

$$
\text { HI }(\%)=\frac{\text { Economic yield }}{\text { Biological yield }} \times 100
$$

Analysis of combining ability was done using OP Stat software (Sheoron et al. 1998) according to Griffing's (1956) method 2 model 1.

\section{RESULT AND DISCUSSION}

Analysis of variance for combining ability was done for twelve yield and yield contributing traits. The estimates revealed that mean square for general combining ability was significant for all the traits studied except for spike length. Significant mean square for GCA for spike length, spikelets/spike, plant height, tillers per plant, flag leaf area and grains per spike was also reported by Kashif and Khaliq (2003). Mean square for SCA was also non significant for spike length while for all other characters it was significant (Table 1). Non significant SCA mean squares for spike length was also reported by Chowdhary et al. (2005). The presence of significant SCA and GCA mean squares for the traits studied suggest the presence of both additive and non additive gene effects in controlling the inheritance of these traits as reported by Seboka et al. 2009. The existence of higher MS due to GCA compared to MS due to SCA for traits as days to maturity, flag leaf area, plant height, number of spikelets per spike, number of grains per spike,
1000 grain weight and harvest index shows that these traits are mostly governed by additive gene action. The involvement of additive gene effects in controlling flag leaf area, plant height, number of grains per spike and 1000 grain weight was previously reported by Chowdhary et al. (2005), Khan et al. (2007) and Dagustu (2008). While for days to $75 \%$ heading, number of productive tillers/ plant, biological yield/plant and grain yield/plant the SCA mean square were significant and higher than GCA mean squares indicating that these traits were mostly controlled by non additive gene action and therefore heterosis breeding can be a suitable strategy for improvement of these traits. Preponderance for non additive gene action for controlling number of productive tillers/plant was reported by Khan et al. (2007).

The mean value of parents along with their GCA effects for different traits (Table 2) indicates a close relationship between mean performance and GCA effects. On the basis of high per se performance and significant GCA effects in desirable direction parental line KAUZ/ALTAR84/3/MILAN/KAUZ/4/ HUITES was identified as best general combiner for number of grains per spike and thousand grain weight. $\mathrm{KFA} / 2^{*} \mathrm{KACHU}$ was responsible for greater number of spikelets per spike. For biological yield per plant and harvest index Raj 4419 acted as superior general combiner. The results obtained for biological yield are in general agreement to the findings of Singh et al. (2012). While PBW 729 and WH 1187 showed superior combining ability for grain yield per plant. For flag leaf area WH 1187 showed superior combining ability. DBW 50 acted as superior combiner for plant height and NIAW 1594 was responsible for early heading and early

Table 1: Analysis of variance for general combining ability (GCA) and specific combining ability (SCA) for different characters in wheat

Mean sum of squares of various characters

\begin{tabular}{|c|c|c|c|c|c|c|c|c|c|c|c|c|c|}
\hline \multirow[b]{2}{*}{$\begin{array}{l}\text { Source of } \\
\text { variation }\end{array}$} & \multicolumn{13}{|c|}{ Mean sum of squares of various characters } \\
\hline & $\underset{ن}{ت}$ & 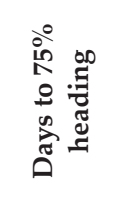 & 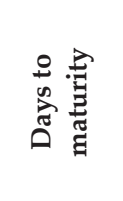 & 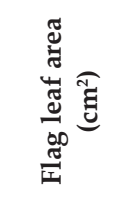 & 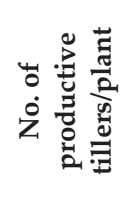 & 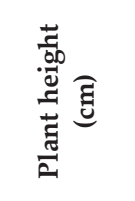 & 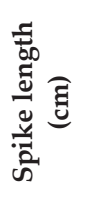 & 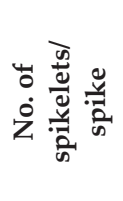 & 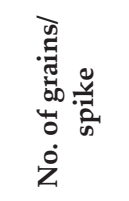 & 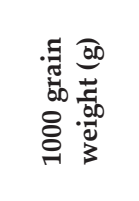 & 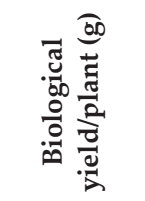 & 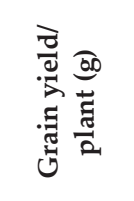 & 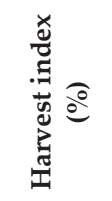 \\
\hline GCA & 9 & $13.606^{* *}$ & $16.748^{* *}$ & $235.777^{* *}$ & $48.250^{* *}$ & $216.807^{* *}$ & 1.690 & $17.131^{* *}$ & $250.055^{* *}$ & $132.741^{* *}$ & $1870.058^{* *}$ & $432.658^{* *}$ & $77.314^{* *}$ \\
\hline
\end{tabular}

** Significant at 1\% probability level. 
Table 2: Estimates of general combining ability (GCA) effects of parents for different traits in wheat

\begin{tabular}{|c|c|c|c|c|c|c|c|c|c|c|c|c|}
\hline Parents & \multicolumn{2}{|c|}{$\begin{array}{c}\text { Days to } 75 \% \\
\text { heading }\end{array}$} & \multicolumn{2}{|c|}{$\begin{array}{l}\text { Days to } \\
\text { maturity }\end{array}$} & \multicolumn{2}{|c|}{$\begin{array}{c}\text { Flag leaf area } \\
\left(\mathrm{cm}^{2}\right)\end{array}$} & \multicolumn{2}{|c|}{$\begin{array}{c}\text { No. of productive } \\
\text { tillers/ plant }\end{array}$} & \multicolumn{2}{|c|}{$\begin{array}{l}\text { Plant height } \\
(\mathrm{cm})\end{array}$} & \multicolumn{2}{|c|}{$\begin{array}{c}\text { Spike length } \\
(\mathrm{cm})\end{array}$} \\
\hline UP 2762 & -0.378 & 93.3 & $0.567^{*}$ & 141.3 & $-3.740^{* *}$ & 23.4 & -0.621 & 22.3 & -0.508 & 84.2 & -0.149 & 12.6 \\
\hline $\mathrm{KFA} / 2^{*} \mathrm{KACHU}$ & $-0.711^{*}$ & 98 & $-1.156^{* *}$ & 141.3 & -1.084 & 21.5 & 1.059 & 21.3 & $2.466^{* *}$ & 87.9 & -0.231 & 11.9 \\
\hline PBW 729 & $0.956^{* *}$ & 99 & $-0.544^{*}$ & 141.7 & -1.557 & 28.2 & 1.739 & 28 & $-2.306^{* *}$ & 86.4 & 0.236 & 12.3 \\
\hline WH 1187 & -0.461 & 94.3 & -0.072 & 141.3 & $3.897^{* *}$ & 34.7 & 0.775 & 15.3 & $-2.572^{* *}$ & 85.6 & -0.249 & 11.5 \\
\hline HD 2967 & $0.650^{*}$ & 93.3 & 0.456 & 140 & 0.175 & 24.7 & 0.614 & 23.2 & $2.595^{* *}$ & 97.2 & -0.237 & 11.9 \\
\hline DBW 50 & 0.456 & 937 & $1.094^{* *}$ & 144 & $-2.307^{*}$ & 24.8 & $-2.407^{*}$ & 23.6 & $-3.855^{* *}$ & 72.4 & -0.070 & 12.8 \\
\hline NIAW 1594 & $-0.961^{* *}$ & 92.3 & $-0.822^{* *}$ & 136 & $3.618^{* *}$ & 32.4 & 0.043 & 19.1 & $2.546^{* *}$ & 93.9 & 0.237 & 13.1 \\
\hline QLD 39 & 0.150 & 93 & 0.094 & 141.7 & 0.702 & 28.5 & -0.804 & 21.9 & $-1.344^{*}$ & 92.3 & 0.166 & 14.3 \\
\hline
\end{tabular}

*,** Significant at $5 \%$ and $1 \%$ probability level, respectively.

\begin{tabular}{|c|c|c|c|c|c|c|c|c|c|c|c|c|}
\hline Parents & \multicolumn{2}{|c|}{$\begin{array}{c}\begin{array}{c}\text { No. of spikelets/ } \\
\text { spike }\end{array} \\
\end{array}$} & \multicolumn{2}{|c|}{$\begin{array}{c}\text { No. of grains/ } \\
\text { spike }\end{array}$} & \multicolumn{2}{|c|}{$\begin{array}{l}1000 \text { grain } \\
\text { weight }(\mathrm{g})\end{array}$} & \multicolumn{2}{|c|}{$\begin{array}{c}\text { Biological yield/ } \\
\text { plant }(\mathrm{g})\end{array}$} & \multicolumn{2}{|c|}{$\begin{array}{c}\text { Grain yield/ plant } \\
(\mathrm{g})\end{array}$} & \multicolumn{2}{|c|}{$\begin{array}{c}\text { Harvest index } \\
(\%)\end{array}$} \\
\hline UP 2762 & 0.060 & 23.2 & $-2.63^{* *}$ & 63.3 & $-2.187^{* *}$ & 40.7 & $-5.254^{*}$ & 154.7 & $-2.827^{* *}$ & 61.0 & $-0.810^{*}$ & 39.3 \\
\hline $\mathrm{KFA} / 2^{*} \mathrm{KACHU}$ & $0.875^{* *}$ & 23.1 & $3.194^{* *}$ & 72.7 & $1.555^{* *}$ & 41.2 & $6.979^{* *}$ & 91.4 & $4.734^{* *}$ & 35.6 & $1.586^{* *}$ & 38.9 \\
\hline PBW 729 & 0.246 & 23.8 & -0.203 & 64.5 & 0.594 & 45.5 & $9.389^{* *}$ & 176.6 & $2.209^{*}$ & 66.0 & $-1.326^{* *}$ & 37.3 \\
\hline WH 1187 & $-1.497^{* *}$ & 17.1 & $1.980^{* *}$ & 76.5 & $0.888^{* *}$ & 44.7 & $4.626^{*}$ & 135.8 & $3.315^{* *}$ & 57.2 & $0.908^{*}$ & 42.0 \\
\hline HD 2967 & -0.594 & 22.5 & $-2.667^{* *}$ & 63 & -0.673 & 44.8 & -1.443 & 107.5 & -0.799 & 43.2 & $-0.719^{*}$ & 39.8 \\
\hline DBW 50 & -0.478 & 23.9 & $-2.409^{* *}$ & 63.4 & $-3.023^{* *}$ & 42.3 & $-9.415^{* *}$ & 135.8 & $-4.841^{* *}$ & 50.4 & -0.372 & 37.2 \\
\hline NIAW 1594 & 0.586 & 24.5 & $2.338^{* *}$ & 69.1 & $1.808^{* *}$ & 45.9 & $10.051^{* *}$ & 107.7 & $4.323^{* *}$ & 47.6 & 0.085 & 44.1 \\
\hline QLD 39 & 0.088 & 24 & $-3.014^{* *}$ & 50.2 & $-2.376^{* *}$ & 32.9 & -1.762 & 116.6 & $-4.146^{* *}$ & 35.0 & $-2.947^{* *}$ & 30.1 \\
\hline
\end{tabular}

*,** Significant at $5 \%$ and $1 \%$ probability level, respectively.

maturity. These lines having significant estimates of GCA in desirable direction along with higher per se performance can be used as parental line in crosses effected with the main aim of accessing improvement of these traits.

The crosses with SCA effects and mean performance have been depicted in table 3 . The crosses having higher SCA effects in desirable direction for different traits can be utilized to produce superior transgressive segregant for these traits. The involvement of parents with good general combining ability for specific traits in certain crosses can be attributed to superior SCA effects of these crosses for these traits. Out of total 45 crosses 5, 8, 4, 7, 8, $3,3,11,11,13,8$ and 11 crosses showed significant SCA effects in desirable direction for days to $75 \%$ heading, days to maturity, flag leaf area, number of productive tillers per plant, plant height, spike length, number of spikelets/spike, number of grains/ spike, 1000 grain weight, biological yield/plant, grain yield/plant and harvest index, respectively. The ranking of specific cross combinations on the basis of their per se performance and SCA effects were computed in Table 4. Grain yield is considered as one of the most important trait from breeding point of view as it decides the success of a particular breeding programme. Five crosses namely, WH 1187 x HD 2967, KFA/2*KACHU x NIAW 1594, $\mathrm{KFA} / 2 * \mathrm{KACHU} \times \mathrm{PBW} 729, \mathrm{WH} 1187 \times$ QLD 39 and HD $2967 \times$ NIAW 1594 showed significant positive SCA effects for grain yield. Crosses with significant and positive SCA effects for grain yield were also 
Table 3: Specific combining ability effects of crosses for various traits in wheat

\begin{tabular}{|c|c|c|c|c|c|c|c|c|c|c|c|c|}
\hline \multirow[t]{2}{*}{ Crosses } & \multicolumn{2}{|l|}{$\begin{array}{c}\text { Days } \\
\text { to } 75 \% \\
\text { heading }\end{array}$} & \multicolumn{2}{|l|}{$\begin{array}{l}\text { Days to } \\
\text { maturity }\end{array}$} & \multicolumn{2}{|l|}{$\begin{array}{l}\text { Flag leaf } \\
\text { area }\left(\mathrm{cm}^{2}\right)\end{array}$} & \multicolumn{2}{|l|}{$\begin{array}{l}\text { No. of } \\
\text { productive } \\
\text { tillers/ } \\
\text { plant }\end{array}$} & \multicolumn{2}{|l|}{$\begin{array}{l}\text { Plant } \\
\text { height } \\
(\mathrm{cm})\end{array}$} & \multicolumn{2}{|l|}{$\begin{array}{l}\text { Spike } \\
\text { length } \\
(\mathrm{cm})\end{array}$} \\
\hline & $\begin{array}{l}\text { SCA } \\
\text { effect }\end{array}$ & $\begin{array}{r}\text { mean } \mathrm{S} \\
\mathrm{e}\end{array}$ & $\begin{array}{l}\text { SCA } \\
\text { effect }\end{array}$ & mean & $\begin{array}{l}\text { SCA } \\
\text { effect }\end{array}$ & mean & $\begin{array}{l}\text { SCA } \\
\text { effect }\end{array}$ & mean & $\begin{array}{l}\text { SCA } \\
\text { effect }\end{array}$ & mean & $\begin{array}{l}\text { SCA } \\
\text { effect }\end{array}$ & mean \\
\hline $\begin{array}{l}\text { KAUZ/ALTAR84/3/MILAN/ } \\
\text { KAUZ/4/HUITES × UP } 2762\end{array}$ & -0.462 & 94.30 & 0.104 & 141.3 & -2.129 & 20.2 & -2.587 & 19.3 & -1.017 & 86.5 & -0.483 & 12.5 \\
\hline $\begin{array}{l}\text { KAUZ/ALTAR84/3/ } \\
\text { MILAN/KAUZ/4/HUITES × } \\
\text { KFA/2*KACHU }\end{array}$ & -1.462 & 93.0 & 0.492 & 140 & 2.656 & 27.6 & 0.909 & 24.5 & 1.675 & 92.2 & -0.285 & 12.6 \\
\hline $\begin{array}{l}\text { KAUZ/ALTAR84/3/MILAN/ } \\
\text { KAUZ/4/HUITES × Raj } 4419\end{array}$ & -0.045 & 95.3 & -0.674 & 140 & -2.316 & 25.8 & 2.348 & 24.5 & 3.547 & 93.9 & 0.148 & 13.3 \\
\hline $\begin{array}{l}\text { KAUZ/ALTAR84/3/MILAN/ } \\
\text { KAUZ/4/HUITES × PBW } 729\end{array}$ & $4.538^{* *}$ & 100.71 & $1.881^{*}$ & 142 & -0.262 & 24.2 & -3.734 & 20.5 & 0.171 & 85.9 & -0.135 & 13.2 \\
\hline $\begin{array}{l}\text { KAUZ/ALTAR84/3/MILAN/ } \\
\text { KAUZ/4/HUITES } \times \text { WH } 1187\end{array}$ & -1.712 & 93.0 & 0.076 & 140.7 & 5.431 & 35.4 & $7.374^{*}$ & 30.7 & -1.683 & 83.8 & 0.287 & 13.2 \\
\hline $\begin{array}{l}\text { KAUZ/ALTAR84/3/MILAN/ } \\
\text { KAUZ/4/HUITES × HD } 2967\end{array}$ & -0.157 & 95.7 & -0.452 & 140.7 & $-7.270^{*}$ & 18.9 & 0.968 & 24.1 & -7.063 & 83.6 & -0.439 & 12.4 \\
\hline $\begin{array}{l}\text { KAUZ/ALTAR84/3/MILAN/ } \\
\text { KAUZ/4/HUITES × DBW } 50\end{array}$ & $4.371^{* *}$ & 100 & $2.242^{*}$ & 144 & 0.705 & 24.4 & -5.945 & 14.2 & $9.930^{* *}$ & 94.1 & 0.391 & 13.4 \\
\hline $\begin{array}{l}\text { KAUZ/ALTAR84/3/MILAN/ } \\
\text { KAUZ/4/HUITES × NIAW } 1594\end{array}$ & 1.788 & 96 & $2.492^{* *}$ & 142.3 & -0.150 & 29.5 & -1.918 & 20.6 & $-5.147^{*}$ & 85.4 & 0.941 & 14.3 \\
\hline $\begin{array}{l}\text { KAUZ/ALTAR84/3/MILAN/ } \\
\text { KAUZ/4/HUITES × QLD } 39\end{array}$ & $-2.990^{* *}$ & 92.3 & $-4.758^{* *}$ & 136 & -4.887 & 21.8 & $-6.958^{*}$ & 14.8 & $-5.125^{*}$ & 81.6 & -0.355 & 12.9 \\
\hline UP $2762 \times$ KFA/2*KACHU & -0.323 & 93.7 & -0.035 & 139.7 & -3.520 & 19.4 & -2.033 & 21 & -4.150 & 85.2 & 0.381 & 12.8 \\
\hline UP 2762 × Raj 4419 & 0.427 & 95.3 & 0.131 & 141 & $8.855^{* *}$ & 34.9 & 4.589 & 26.2 & 3.869 & 93.1 & 0.400 & 13.1 \\
\hline UP $2762 \times$ PBW 729 & -1.657 & 94 & 1.354 & 141.7 & 2.719 & 25.2 & $-7.279^{*}$ & 16.4 & $-4.611^{*}$ & 80 & 0.347 & 13.3 \\
\hline UP $2762 \times$ WH 1187 & -0.240 & 94 & 0.881 & 141.7 & -2.611 & 25.3 & -0.749 & 22 & -0.731 & 83.6 & 0.649 & 13.1 \\
\hline UP $2762 \times$ HD 2967 & -0.684 & 94.7 & $-2.980^{* *}$ & 138.3 & -4.469 & 19.7 & 1.079 & 23.7 & $13.098^{* *}$ & 102.6 & $-1.407^{* *}$ & 11.4 \\
\hline UP $2762 \times$ DBW 50 & -0.490 & 94.7 & 0.048 & 142 & -3.570 & 18.2 & $8.890^{* *}$ & 28.4 & -0.518 & 82.5 & 0.543 & 13.2 \\
\hline UP $2762 \times$ NIAW 1594 & 1.593 & 95.3 & -0.369 & 139.7 & 0.451 & 28.1 & -0.684 & 21.3 & 2.771 & 92.2 & -0.964 & 12 \\
\hline UP $2762 \times$ QLD 39 & $3.816^{* *}$ & 98.7 & 1.048 & 142 & -1.885 & 22.9 & -3.073 & 18.1 & -4.230 & 81.3 & 0.040 & 12.9 \\
\hline KFA/2*KACHU × Raj 4419 & -1.907 & 92.7 & 0.854 & 140 & -1.794 & 26.9 & $9.243^{* *}$ & 32.6 & -2.586 & 89.7 & 0.591 & 13.2 \\
\hline $\mathrm{KFA} / 2^{*} \mathrm{KACHU} \times \mathrm{PBW} 729$ & -0.990 & 94.3 & -0.924 & 137.7 & 1.947 & 27.1 & $6.618^{*}$ & 32 & $-5.075^{*}$ & 82.5 & $1.245^{*}$ & 14.1 \\
\hline $\mathrm{KFA} / 2 * \mathrm{KACHU} \times \mathrm{WH} 1187$ & -1.573 & 92.3 & 0.270 & 139.3 & -2.270 & 28.3 & -1.785 & 22.6 & 0.251 & 87.6 & -0.362 & 12 \\
\hline \multirow[t]{2}{*}{ Crosses } & $\begin{array}{c}\text { Days } \\
\text { to } 75 \% \\
\text { heading }\end{array}$ & & $\begin{array}{l}\text { Days to } \\
\text { maturity }\end{array}$ & & $\begin{array}{l}\text { Flag leaf } \\
\text { area }\left(\mathrm{cm}^{2}\right)\end{array}$ & & $\begin{array}{c}\text { No. of } \\
\text { productive } \\
\text { tillers/ } \\
\text { Plant }\end{array}$ & & $\begin{array}{l}\text { Plant } \\
\text { height } \\
(\mathrm{cm})\end{array}$ & & $\begin{array}{l}\text { Spike } \\
\text { length } \\
(\mathrm{cm})\end{array}$ & \\
\hline & $\begin{array}{l}\text { SCA } \\
\text { effect }\end{array}$ & Mean & $\begin{array}{l}\text { SCA } \\
\text { effect }\end{array}$ & mean & $\begin{array}{l}\text { SCA } \\
\text { effect }\end{array}$ & mean & SCA effect & mean & $\begin{array}{l}\text { SCA } \\
\text { effect }\end{array}$ & mean & $\begin{array}{l}\text { SCA } \\
\text { effect }\end{array}$ & mean \\
\hline KFA/2*KACHU $\times$ HD 2967 & 0.316 & 95.3 & 0.409 & 140 & 3.039 & 29.9 & -3.157 & 21.1 & 0.747 & 93.2 & -0.309 & 12.1 \\
\hline $\mathrm{KFA} / 2^{*} \mathrm{KACHU} \times \mathrm{DBW} 50$ & -1.823 & 93 & $-4.563^{* *}$ & 135.7 & 3.914 & 28.3 & 0.420 & 21.7 & $6.130^{* *}$ & 92.2 & 0.431 & 13 \\
\hline KFA/2*KACHU × NIAW 1594 & -1.073 & 92.3 & -0.646 & 137.7 & 5.799 & 36.1 & 0.613 & 24.3 & $5.407^{*}$ & 97.8 & 0.048 & 12.9 \\
\hline $\mathrm{KFA} / 2 * \mathrm{KACHU} \times \mathrm{QLD} 39$ & 0.149 & 94.7 & $-2.563^{* *}$ & 136.7 & -1.488 & 25.9 & -4.087 & 18.8 & $6.562^{* *}$ & 95.1 & -0.778 & 12 \\
\hline Raj $4419 \times$ PBW 729 & $3.427^{* *}$ & 99.7 & $2.909^{* *}$ & 142.7 & -5.278 & 23 & $-7.390^{*}$ & 16.6 & $-5.757^{* *}$ & 81.7 & 0.368 & 13.4 \\
\hline Raj $4419 \times$ WH 1187 & $3.843^{* *}$ & 98.7 & 0.437 & 140.7 & -3.622 & 30.1 & 0.197 & 23.2 & $-5.211^{*}$ & 82 & 0.010 & 12.6 \\
\hline Raj 4419 × HD 2967 & -0.601 & 95.3 & -0.758 & 140 & 5.327 & 35.3 & 1.025 & 23.9 & $7.676^{* *}$ & 100.1 & 0.280 & 12.9 \\
\hline Raj 4419 × DBW 50 & $-2.407^{*}$ & 93.3 & -0.396 & 141 & 0.759 & 28.3 & 1.255 & 21.1 & $7.036^{* *}$ & 93 & 0.497 & 13.3 \\
\hline Raj 4419 × NIAW 1594 & -0.323 & 94 & 1.187 & 140.7 & -0.110 & 33.3 & $-6.195^{*}$ & 16.1 & -2.452 & 89.9 & -0.400 & 12.7 \\
\hline Raj 4419 × QLD 39 & -1.434 & 94 & -1.063 & 139.3 & $7.544^{*}$ & 38.1 & -1.461 & 20 & -3.342 & 85.1 & 0.161 & 13.2 \\
\hline PBW $729 \times$ WH 1187 & $-3.573^{* *}$ & 92 & $-3.674^{* *}$ & 136 & -4.244 & 25.9 & -3.798 & 21.3 & 1.333 & 83.9 & 0.147 & 13 \\
\hline PBW $729 \times$ HD 2967 & -1.351 & 95.3 & 0.465 & 140.7 & 3.701 & 30.1 & 0.430 & 25.4 & 0.296 & 88 & 0.918 & 13.8 \\
\hline
\end{tabular}


PBW $729 \times$ DBW 50

$\begin{array}{llllllllllll}-3.490^{* *} & 93 & -6.174^{* *} & 134.7 & -0.624 & 23.3 & -3.826 & 18.1 & 3.079 & 84.3 & -0.242 & 12.8 \\ -0.073 & 95 & 2.409^{* *} & 141.3 & -4.725 & 25.1 & 20.057^{* *} & 44.4 & 2.569 & 90.2 & 0.218 & 13.5 \\ -0.851 & 95.3 & -3.174^{* *} & 136.7 & -0.325 & 26.6 & -5.019 & 18.5 & 0.735 & 84.5 & -0.789 & 12.4 \\ 0.399 & 95.7 & 0.659 & 141.3 & 6.644^{*} & 38.5 & 3.527 & 27.5 & -2.601 & 84.8 & 1.043^{*} & 13.4 \\ 4.927^{* *} & 100 & 0.020 & 141.3 & 0.893 & 30.3 & 0.704 & 21.7 & 3.405 & 84.4 & 0.430 & 12.9 \\ -2.990^{* *} & 90.7 & -3.396^{* *} & 136 & 7.521^{*} & 42.8 & -2.313 & 21.1 & 1.395 & 88.8 & -0.454 & 12.4 \\ 0.566 & 95.3 & 2.354^{* *} & 142.7 & -5.902 & 26.5 & 14.444^{* *} & 37 & -0.829 & 82.7 & -0.083 & 12.7 \\ 4.816^{* *} & 101 & 3.159^{* *} & 145 & -4.042 & 21.6 & -15.791^{* *} & 5.0 & -13.988^{* *} 72.2 & -1.526^{* *} & 11 \\ 0.232 & 95 & 1.076 & 141 & -1.917 & 29.7 & 6.025 & 29.3 & -1.122 & 91.4 & 1.307^{*} & 14.1 \\ 3.121^{* *} & 99 & 0.826 & 141.7 & 5.943 & 34.6 & 7.072^{*} & 29.5 & -6.233^{* *} & 82.4 & 0.604 & 13.4 \\ -0.573 & 94 & 0.104 & 140.7 & -2.619 & 26.5 & -1.564 & 18.7 & -1.972 & 84.1 & -0.056 & 12.9 \\ -0.684 & 95 & 2.520^{* *} & 144 & 1.411 & 27.6 & 4.159 & 23.5 & 1.440 & 83.7 & -0.729 & 12.2 \\ 3.066^{* *} & 97.3 & 2.437^{* *} & 142 & 1.003 & 33.1 & -6.857^{*} & 15 & -4.170 & 84.4 & -0.246 & 13 \\ 1.056 & & 0.903 & & 3.248 & & 3.140 & & 2.210 & & 0.530 & \end{array}$

PBW $729 \times$ NIAW 1594

PBW $729 \times$ QLD 39

WH $1187 \times$ HD 2967

WH $1187 \times$ DBW 50

WH $1187 \times$ NIAW 1594

WH $1187 \times$ QLD 39

HD $2967 \times$ DBW 50

HD $2967 \times$ NIAW 1594

HD $2967 \times$ QLD 39

DBW $50 \times$ NIAW 1594

DBW $50 \times$ QLD 39

NIAW $1594 \times$ QLD 39

1.056

0.903

*, ** Significant at 5\% and 1\% probability level, respectively.

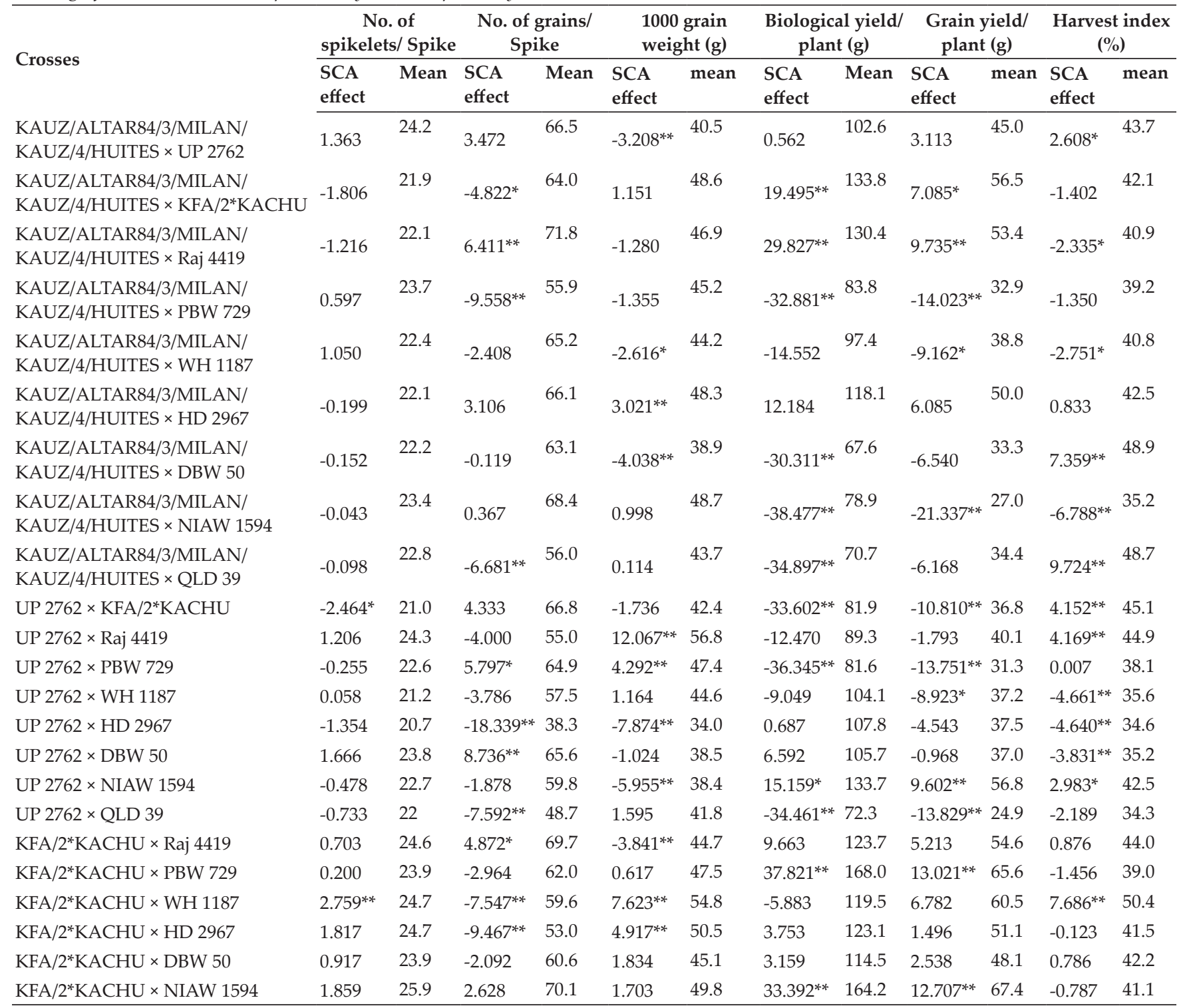


Combining ability analysis for yield and yield contributing traits in bread

\begin{tabular}{|c|c|c|c|c|c|c|c|c|c|c|c|c|}
\hline \multirow{2}{*}{ Crosses } & \multicolumn{2}{|c|}{$\begin{array}{c}\text { No. of spikelets/ } \\
\text { Spike } \\
\end{array}$} & \multicolumn{2}{|c|}{$\begin{array}{l}\text { No. of grains/ } \\
\text { Spike }\end{array}$} & \multicolumn{2}{|c|}{$\begin{array}{l}1000 \text { grain } \\
\text { weight }(\mathrm{g})\end{array}$} & \multicolumn{2}{|c|}{$\begin{array}{c}\text { Biological yield/ } \\
\text { plant (g) }\end{array}$} & \multicolumn{2}{|c|}{$\begin{array}{l}\text { Grain yield/ } \\
\text { plant (g) }\end{array}$} & \multicolumn{2}{|c|}{$\begin{array}{c}\text { Harvest index } \\
(\%)\end{array}$} \\
\hline & $\begin{array}{l}\text { SCA } \\
\text { effect }\end{array}$ & mean & $\begin{array}{l}\text { SCA } \\
\text { effect }\end{array}$ & mean & $\begin{array}{l}\text { SCA } \\
\text { effect }\end{array}$ & Mean & $\begin{array}{l}\text { SCA } \\
\text { effect }\end{array}$ & Mean & $\begin{array}{l}\text { SCA } \\
\text { effect }\end{array}$ & Mean & $\begin{array}{l}\text { SCA } \\
\text { effect }\end{array}$ & mean \\
\hline Raj $4419 \times$ PBW 729 & -0.760 & 22.5 & $-6.064^{* *}$ & 55.4 & $-2.713^{*}$ & 44.8 & $-38.930^{* *}$ & 77.5 & $-17.596^{* *}$ & 29.3 & $-2.665^{*}$ & 37.6 \\
\hline Raj $4419 \times$ WH 1187 & 1.063 & 22.6 & 1.219 & 64.8 & 1.559 & 49.4 & 3.349 & 115.0 & -1.235 & 46.8 & $-2.433^{*}$ & 40.1 \\
\hline Raj $4419 \times$ NIAW 1594 & -0.977 & 22.6 & -4.006 & 60.0 & $-3.194^{* *}$ & 45.6 & -10.076 & 107.0 & -6.843 & 42.2 & $-2.350^{*}$ & 39.3 \\
\hline Raj 4419 × QLD 39 & 0.884 & 24 & -0.319 & 58.3 & $3.256^{* *}$ & 47.8 & -11.629 & 93.7 & -1.840 & 38.7 & $2.669^{*}$ & 41.3 \\
\hline PBW $729 \times$ WH 1187 & -0.681 & 20.6 & -1.017 & 62.7 & $-5.083^{* *}$ & 41.1 & $-36.926^{* *}$ & 90.9 & $-15.626^{* *}$ & 35.6 & -0.692 & 39.1 \\
\hline PBW $729 \times$ HD 2967 & 1.329 & 23.5 & $12.031^{* *}$ & 71.1 & -0.588 & 44.1 & 5.744 & 127.5 & $8.488^{*}$ & 55.6 & $4.715^{* *}$ & 43.4 \\
\hline PBW 729 × DBW 50 & -0.357 & 22 & $-6.294^{* *}$ & 53.0 & 1.562 & 43.9 & -5.618 & 108.1 & 0.129 & 43.2 & 1.281 & 39.8 \\
\hline WH 1187 × NIAW 1594 & -0.285 & 21.4 & 0.175 & 66.4 & $-3.963^{* *}$ & 43.5 & $-25.722^{* *}$ & 102.7 & $-11.640^{* *}$ & 41.7 & -0.613 & 40.6 \\
\hline WH 1187 × QLD 39 & -1.130 & 20 & -3.539 & 57.4 & -0.780 & 42.5 & $41.992^{* *}$ & 158.6 & $20.129^{* *}$ & 65.0 & $2.635^{*}$ & 40.8 \\
\hline HD $2967 \times$ DBW 50 & $-8.213^{* *}$ & 13.3 & $-18.964^{* *}$ & 37.9 & $-9.238^{* *}$ & 31.8 & $-81.352^{* *}$ & 21.6 & $-30.529^{* *}$ & 9.5 & $4.404^{* *}$ & 44.1 \\
\hline HD $2967 \times$ NIAW 1594 & -1.281 & 21.3 & $6.489^{* *}$ & 68.1 & 0.964 & 46.8 & $24.714^{* *}$ & 147.1 & $11.640^{* *}$ & 60.8 & 1.185 & 41.3 \\
\hline HD 2967 × QLD 39 & -0.066 & 22 & $7.308^{* *}$ & 63.6 & $4.848^{* *}$ & 46.5 & $21.662^{* *}$ & 132.2 & 2.577 & 43.3 & $-4.427^{* *}$ & 32.7 \\
\hline DBW $50 \times$ NIAW 1594 & -0.117 & 22.6 & $-5.536^{*}$ & 56.3 & 1.448 & 45.0 & $15.087^{*}$ & 129.5 & 6.749 & 51.9 & 0.100 & 40.0 \\
\hline DBW 50 × QLD 39 & 1.811 & 24 & $11.117^{* *}$ & 67.6 & -1.836 & 37.5 & -2.466 & 100.1 & -1.182 & 35.5 & -1.635 & 35.3 \\
\hline NIAW 1594 × QLD 39 & -0.017 & 23.2 & -0.231 & 61.0 & $7.367^{* *}$ & 51.5 & 10.734 & 132.8 & 4.321 & 50.2 & 0.269 & 37.6 \\
\hline SE (ij) & 1.060 & & 2.326 & & 1.157 & & 7.549 & & 3.588 & & 1.187 & \\
\hline
\end{tabular}

*,** Significant at $5 \%$ and $1 \%$ probability level, respectively.

Table 4: Ranking of good cross combination on the basis of per se performance and their SCA effect in a $10 \times 10$ diallel cross of wheat

\begin{tabular}{|c|c|c|c|}
\hline Character & Parent with higher per se performance & Good cross combination & Superior common cross combination \\
\hline \multirow{5}{*}{$\begin{array}{l}\text { Days to } 75 \% \\
\text { heading }\end{array}$} & 1. WH $1187 \times$ NIAW 1594 & 1. PBW $729 \times$ WH 1187 & PBW $729 \times$ WH 1187 \\
\hline & 2. PBW $729 \times$ WH 1187 & 2. $\mathrm{PBW} 729 \times \mathrm{DBW} 50$ & WH $1187 \times$ NIAW 1594 \\
\hline & 3. KFA/2*KACHU $\times$ NIAW 1594 & 3. WH $1187 \times$ NIAW 1594 & \multirow{3}{*}{$\begin{array}{l}\text { KAUZ/ALTAR84/3/MILAN/KAUZ/4/ } \\
\text { HUITES } \times \text { QLD } 39\end{array}$} \\
\hline & 5. KAUZ/ALTAR84/3/MILAN/KAUZ/4/ & HUITES × QLD 39 & \\
\hline & HUITES $\times$ QLD 39 & 5. Raj $4419 \times$ DBW 50 & \\
\hline \multirow{4}{*}{$\begin{array}{l}\text { Days to } \\
\text { maturity }\end{array}$} & 3. PBW $729 \times$ WH 1187 & HUITES × QLD 39 & \multirow{4}{*}{$\begin{array}{l}\text { PBW } 729 \times \text { DBW } 50 \\
\text { KFA/2*KACHU × DBW } 50 \\
\text { PBW } 729 \times \text { WH } 1187 \\
\text { WH } 1187 \times \text { NIAW } 1594\end{array}$} \\
\hline & 4. WH $1187 \times$ NIAW 1594 & 3. KFA/2*KACHU × DBW 50 & \\
\hline & 5. PBW $729 \times$ QLD 39 & 4. PBW $729 \times$ WH 1187 & \\
\hline & & 5. WH $1187 \times$ NIAW 1594 & \\
\hline \multirow[t]{2}{*}{ Flag leaf area } & 1. WH $1187 \times$ NIAW 1594 & 1. UP $2762 \times$ Raj 4419 & \multirow{2}{*}{$\begin{array}{l}\text { Raj } 4419 \times \text { QLD } 39 \\
\text { WH } 1187 \times \text { NIAW } 1594 \\
\text { WH } 1187 \times \text { HD } 2967\end{array}$} \\
\hline & $\begin{array}{l}\text { 5. KAUZ/ALTAR84/3/MILAN/KAUZ/4/ } \\
\text { HUITES } \times \text { WH } 1187\end{array}$ & & \\
\hline \multirow{5}{*}{$\begin{array}{l}\text { Productive } \\
\text { tillers/plant }\end{array}$} & 1. PBW $729 \times$ NIAW 1594 & 1. PBW $729 \times$ NIAW 1594 & PBW $729 \times$ NIAW 1594 \\
\hline & 2. WH $1187 \times$ QLD 39 & 2. WH $1187 \times$ QLD 39 & WH 1187 × QLD 39 \\
\hline & 3. KFA/2*KACHU × Raj 4419 & 3. KFA/2*KACHU $\times$ Raj 4419 & KFA/2*KACHU × Raj 4419 \\
\hline & 4. KFA/2*KACHU × PBW 729 & 4. UP $2762 \times$ DBW 50 & \multirow{2}{*}{$\begin{array}{l}\text { KAUZ/ALTAR84/3/MILAN/KAUZ/4/ } \\
\text { HUITES } \times \text { WH } 1187\end{array}$} \\
\hline & $\begin{array}{l}\text { 5. KAUZ/ALTAR84/3/MILAN/KAUZ/4/ } \\
\text { HUITES } \times \text { WH } 1187\end{array}$ & $\begin{array}{l}\text { 5. KAUZ/ALTAR84/3/MILAN/KAUZ/4/ } \\
\text { HUITES } \times \text { WH } 1187\end{array}$ & \\
\hline
\end{tabular}




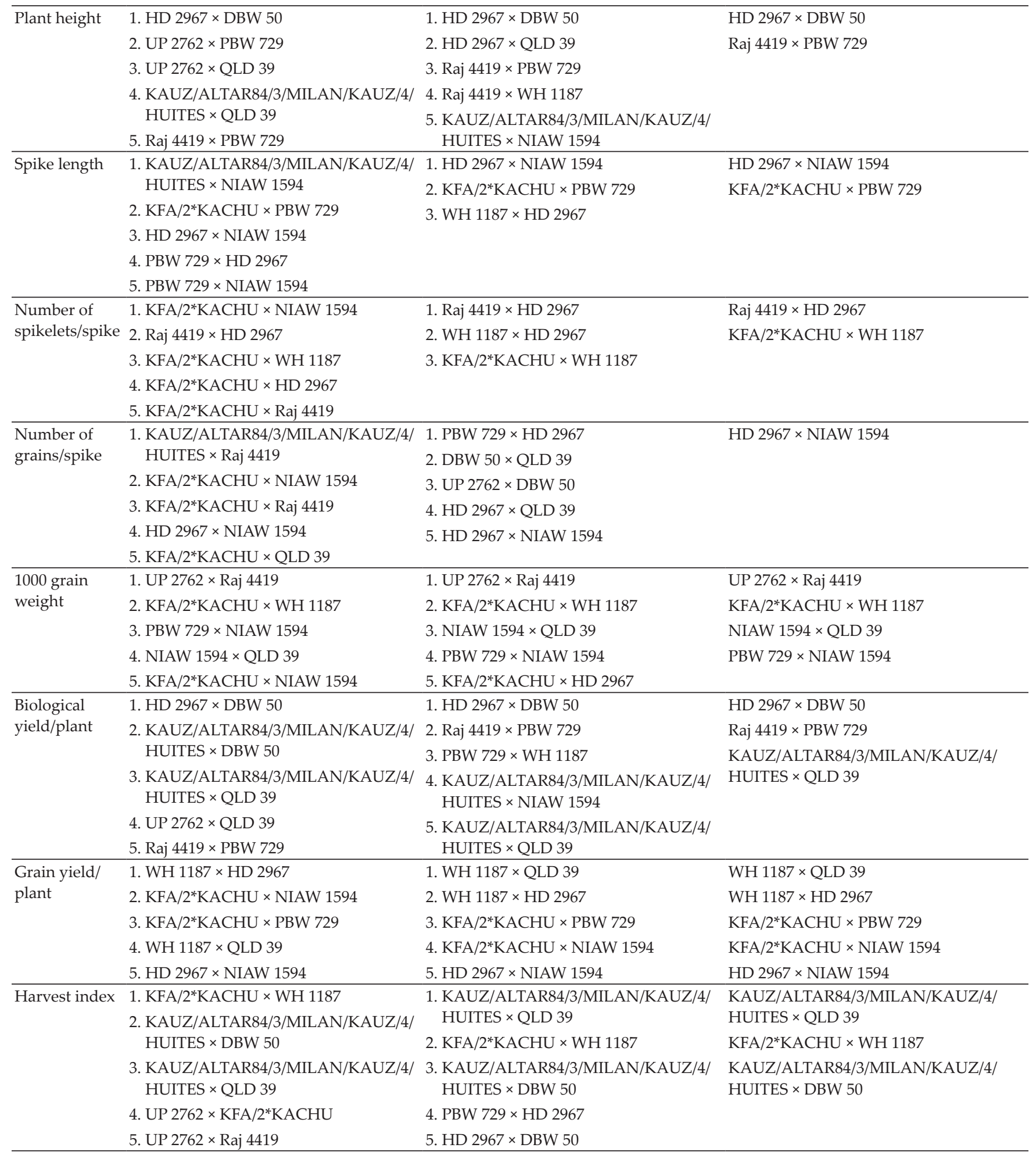

identified by Joshi et al. (2016). However on the basis of both per se performance and significant positive SCA effect crosses WH $1187 \times$ QLD 39, WH $1187 \times$ HD 2967, KFA/2*KACHU × PBW 729, $\mathrm{KFA} / 2 * \mathrm{KACHU} \times \mathrm{NIAW} 1594$ and HD $2967 \times$ NIAW 1594 were identified as superior crosses for producing desirable trangressive segregant for grain yield. Similarly on basis of good mean performance and significant SCA effect common superior crosses for days to $75 \%$ heading were PBW $729 \times \mathrm{WH} 1187$, WH $1187 \times$ NIAW 1594, KAUZ/ALTAR84/3/MILAN/ KAUZ/4/HUITES $\times$ QLD 39, for days to maturity were PBW $729 \times \mathrm{DBW} 50, \mathrm{KFA} / 2 * \mathrm{KACHU} \times \mathrm{DBW}$ 50, PBW $729 \times$ WH 1187, WH $1187 \times$ NIAW 1594, 
for flag leaf area were Raj $4419 \times$ QLD 39, WH 1187 $\times$ NIAW 1594, WH $1187 \times$ HD 2967, for number of productive tillers/plant were PBW $729 \times$ NIAW 1594, WH 1187 x QLD 39, KFA/2*KACHU x Raj 4419, KAUZ/ALTAR84/3/MILAN/KAUZ/4/HUITES x WH 1187, for plant height were HD $2967 \times$ DBW 50, Raj $4419 \times$ PBW 729, for spike length were HD $2967 \times$ NIAW 1594, KFA/2*KACHU $\times$ PBW 729, for number of spikelets/spike were Raj $4419 \times$ HD 2967, $\mathrm{KFA} / 2{ }^{*} \mathrm{KACHU} \times \mathrm{WH} 1187$, for number of grains/ spike were HD $2967 \times$ NIAW 1594, for 1000 grain weight were UP $2762 \times$ Raj 4419, KFA/2*KACHU × WH 1187, NIAW $1594 \times$ QLD 39, PBW $729 \times$ NIAW 1594, for biological yield/plant were HD $2967 \times$ DBW 50, Raj 4419 x PBW 729, KAUZ/ALTAR84/3/ MILAN/KAUZ/4/HUITES $\times$ QLD 39, for grain yield per plant were WH $1187 \times$ QLD 39, WH $1187 \times$ HD 2967, KFA/2*KACHU $\times$ PBW 729, KFA/2*KACHU $\times$ NIAW 1594, HD $2967 \times$ NIAW 1594 and for harvest index were KAUZ/ALTAR84/3/MILAN/KAUZ/4/ HUITES $\times$ QLD 39, KFA/2*KACHU $\times$ WH 1187, KAUZ/ALTAR84/3/MILAN/KAUZ/4/HUITES $\times$ DBW 50. Some crosses had significant SCA effect and superior per se performance for more than one characters as PBW $729 \times$ WH 1187 acted as good cross combination for two traits i.e., days to $75 \%$ heading and days to maturity, WH $1187 \times$ NIAW 1594 for three traits viz. days to $75 \%$ heading, days to maturity and flag leaf area. KAUZ/ALTAR84/3/ MILAN/KAUZ/4/HUITES × QLD 39 was good specific combiner for three traits namely, days to $75 \%$ heading, biological yield and harvest index, WH $1187 \times$ HD 2967 for two trait i.e. flag leaf area and grain yield/plant, PBW $729 \times$ NIAW 1594 for two trait i.e., plant tillers/plant and thousand grain weight, WH $1187 \times$ QLD 39 for two trait productive tillers/plant and grain yield/plant, HD $2967 \times$ DBW 50 for two trait plant height and biological yield/ plant, Raj $4419 \times$ PBW 729 for two traits viz., plant height and biological yield/plant, KFA/2*KACHU $\times$ PBW 729 for two traits spike length and grain yield/plant, HD $2967 \times$ NIAW 1594 for three traits i.e., spike length, grain yield/plant and number of grains/spike and KFA/2*KACHU $\times$ WH 1187 for three traits viz., (Number of spikelets/spike, thousand grain weight and harvest index.

\section{CONCLUSION}

The study revealed the existence of significant amount of variability amongst parental lines and crosses for almost all the traits studied except for spike length for which both GCA and SCA mean squares were insignificant. This shows that the improvement for all the traits except for spike length can be achieved by means of selection of genotypes with superior traits or by islolation of transgressive segregants. NIAW 1594 was best general combiner for both days to $75 \%$ heading and days to maturity, hence it can be used for development of lines with early maturity. For improvement of yield contributing traits as number of grains/spike and thousand grain weight KAUZ/ ALTAR84/3/MILAN/KAUZ/4/HUITES can be used as one of the parent in hybridisation programme. Two cross combinations viz., HD $2967 \times$ NIAW 1594 and $\mathrm{KFA} / 2^{*} \mathrm{KACHU} \times \mathrm{WH} 1187$ were identified as good crosses due to them having higher per se performance and significant SCA effects for three yield and yield contributing traits so these crosses presents an opportunity for commercial exploitation either in form of hybrid varieties or as base material for selection of potential homozygous lines from transgressive segregants for improvement of yield levels of bread wheat.

\section{REFERENCES}

Anonymous, 2016. Progress Report of All India Coordinated Wheat and Barley Improvement Project, 2015-16, Director's Report. Ed. G.P. Singh, ICAR-Indian Institute of Wheat and Barley Research, Karnal, India. p 96.

Breiman, A. and Graur, D. 1995. Wheat evolution. Israel Journal of Plant Sciences, 43(2): 85-98.

Chowdhry, M.A., Saeed, M.S., Khaliq, I. and Ahsan, M. 2005. Combining ability analysis for some polygenic traits in a $5 \times 5$ diallel cross of bread wheat (Triticum aestivum L. em. Thell). Asian Journal of Plant Sciences, 4(4): 405-408.

Dagustu, N. 2008. Genetic Analysis of Grain Yield per Spike and Some Agronomic Traits in Diallel Crosses of Bread Wheat (Triticum aestivum L. em. Thell). Turk. J. Agric. For., 32: 249-258.

Griffing, B. 1956. Concept of general and specific combining ability in relation to diallel crossing systems. Aust. J. Biol. Sci., 9: 463-493.

Habtamu, S., Amsalu, A. and Habtamu, Z. 2009. Combining Ability Analysis for Bread Wheat (Triticum Aestivum L.). East Africa Journal of Sciences, 3: 87-94.

Joshi, A., Kumar, A., Budhlakoti, V., and Bhatt, N. 2019. Heterosis for quality traits in wheat. International Journal of Chemical Studies, 7(6): 404-408.

Joshi, A., Kumar, A., Budhlakoti, V., and Bhatt, N. 2020. Genetic Analysis of Quality Traits in Bread Wheat. International Journal of Current Microbiology and Applied Sciences, 9(2): 929-938. 
antanex

Joshi, A., Kumar, A., Budhlakoti, V., Bhatt, N., and Prasad, B. 2018a. Analysis of variance and estimation of genetic variability parameters for quality traits in bread wheat (Triticum aestivum L. em. Thell) genotypes. International Journal of Chemical Studies, 6(5): 369-372.

Joshi, A., Kumar, A., Budhlakoti, V., Bhatt, N., and Tabassum. 2018 b. Estimation of genetic variability parameters for yield and its components in bread wheat (Triticum aestivum L. em. Thell) genotypes. Journal of Pharmacognosy and Phytochemistry, SP5: 87-90.

Joshi, S.K., Sharma, S.N., Singhania, D.L. and Sain, R.S. 2016. Genetic analysis of yield and its component traits in spring wheat. Acta Agronomica Hungarica, 51(22): 178-195.

Khan, M.A., Ahmad, N., Akbar, M., Rehman, A., and Iqbal, M.M. 2007. Combining ability analysis in wheat. Pak. J. Agri. Sci., 44(1): 1-5.

Kumar, A., Mishra, V.K., Vyas, R.P. and Singh, V. 2011. Heterosis and combining ability analysis in bread wheat (Triticum aestioum L.). Journal of Plant Breeding and Crop Science, 3(10): 209-217.
Sheoran, O.P., Tonk, D.S., Kaushik, L.S., Hasija, R.C. and Pannu, R.S. 1998. Statistical Software Package for Agricultural Research Workers. Recent Advances in information theory, Statistics \& Computer Applications by D.S. Hooda \& R.C. Hasija Department of Mathematics Statistics, CCS HAU, Hisar (139-143).

Singh, A., Kumar, A., Ahmad, E., Swati and Jaiswal, J. P. 2012. Combining ability and gene action studies for seed yield, its components and quality traits in bread wheat (Triticum aestivum L. em. Thell.). Electronic Journal of Plant Breeding, 3(4): 964-972.

Skovmand, B. and Reynolds, M.P. 2000. Increasing yield potential for marginal areas by exploring genetic resources collections. The Eleventh Regional Wheat Workshop for Eastern, Central and Southern Africa. Addis Ababa, Ethiopia, 18(22): 67-77. 